INTERNATIONAL JOURNAL FOR NUMERICAL AND ANALYTICAL METHODS IN GEOMECHANICS, VOL. 18, 253-278 (1994)

\title{
CLoE, A NEW RATE-TYPE CONSTITUTIVE MODEL FOR GEOMATERIALS THEORETICAL BASIS AND IMPLEMENTATION
}

\author{
RENÉ CHAMBON, JACQUES DESRUES AND WALID HAMMAD \\ Laboratoire 3S-IMG, BP 53X, 3804I Grenoble Cedex, France \\ ROBERT CHARLIER \\ Université de Liége, MSM, 6 quai Baming, 4000 Liège Belgium
}

\section{SUMMARY}

The paper describes the basic ideas and the main features of a new class of constitutive laws, in the framework of incrementally non-linear constitutive equations. CLoE is a generic name for that new class of laws, with reference to consistency at the limit surface, and explicit localization analysis. A top-down analysis of the model is presented, and illustrated by examples.

\section{INTRODUCTION}

Constitutive models for geomaterials are often based on the concepts of the mathematical theory of plasticity. ${ }^{1}$ The numerous variations of the Cam Clay model, and other elastoplastic models sometimes refered as flow theory of plasticity (Drucker-Prager, Mohr-Coulomb models; multipotential models like Lade's, Vermeer's, ...) belong to that class of models, hereafter called 'classical elastoplasticity'. Besides classical elastoplasticity, another kind of rate-type constitutive equations, incrementally non-linear, have been elaborated in the past 20 years for geomaterials. As an alternative to the elastoplastic framework - characterized among other features by the concepts of separation of strain rate in elastic and plastic parts, strain rates deriving from elastic and plastic potentials - these models have proved to be of real interest especially when a close reproduction of experimental complexity of soil behaviour is required. Since the time of their introduction, these have been used in the Finite Element Method ${ }^{2,3}$ but some difficulties have been encountered sometimes, especially when the stress state approaches the limit surface. ${ }^{3}$ Some drawbacks of incremental constitutive equations have been clarified, ${ }^{3,4}$ but no useful general rules have been stated to avoid them. Moreover, the complexity of the equations often discourages a critical analysis; then it is very difficult to be sure that such equations are free of any internal contradiction. This question cannot be avoided if one wishes to establish the validation of the equations for practical use. Two classes of problems are of special interest:

(1) effectiveness of the equations in Finite element method computations, which means robustness of equations and algorithms and assesment of the reliability of the prediction; 
(2) objectivity and generality of the bifurcation analysis to be performed on the equations if shear band strain localization problems are considered; such problems are among the most topical and challenging that soil modellers have to face.

These two aims have been the essential guidelines in the development of CLoE as reflected in the name itself, which is formed on the words consistency and explicit localization analysis.

This paper is the first of a set of three, each devoted respectively, to the:

(1) Analysis of the model and assessment of its response in homogeneous modelling;

(2) bifurcation analysis;

(3) implementation in Finite Element Method codes and numerical analysis.

In the present paper, Section 2 is devoted to a review of the basic material properties of granular materials, together with a discussion of the way CLoE accounts for each specific item (or does not); then Section 3 aims to lay down basic mathematical requirements for the law. This section can be viewed as a kind of check list in the spirit of Reference 5, with the objective to provide criterions to be met in the perspective of a practical use of the law in a numerical code. All the conditions exhibited are necessary ones but there is no insurance that they are sufficient. When not meeting these conditions, however, models suffer lack of consistency and some problems are likely to arrive when using them.

Section 4 presents in detail, a new class of incrementally non-linear constitutive equations involving interpolations - the CLoE laws. We start from a chosen mathematical form for the stress rate-strain rate relation. Then we discuss how this form can be specified to be a reasonable representation of a real material. We show that a complete determination of the constitutive equations can be obtained on the basis of axisymmetric triaxial responses, - we shall call it the 'basic paths'-and a few complementary material characteristics, including shear banding. The consistency requirements discussed previously lead this class of models to a set of equations called consistency conditions.

The last objective of the present paper is to present in Section 5 the implementation of the model for Hostun Sand 'RF' and to discuss some examples of the response.

As far as strain localization is concerned, although this is the subject of a next paper, it must be mentioned here that a basic idea of the model is to identify the non-linear shear moduli on localization experiments in relation to a non-restrictive non-linear bifurcation analysis. ${ }^{6}$

\section{A REVIEW OF THE BASIC MATERIAL PROPERTIES OF GRANULAR MATERIALS}

Granular materials behave in a merely specific way with respect to other engineering materials. Among the specific features of the behaviour of these materials, some have to be incorporated in any realistic model, and some may be included or not depending on the actual problem to be modelled. An exhaustive model is impossible to make, at least for practical use in finite element codes. In this section, a few important aspects of the real nature of granular material are discussed, and the way CLoE accounts for each of them is indicated.

\subsection{Granular media and continum mechanics}

Granular materials are considered here as continuum media; the discrete nature of granular assemblies is not directly considered when formulating constitutive equations; in this framework, however, the specifity of the behaviour of these materials, due to their granular nature, is taken into account in a continuum approach as far as the formulation of constitutive equations is made 
on the basis of realistic experimental responses. CLoE laws are based on a direct formulation of a set of so-called 'basic paths', hence it allows a close formulation of experimental responses.

\subsection{Dilatancy}

Granular materials exhibit dilatancy (or contractancy). Pure deviatoric stress increments applied to a homogeneous specimen in an elementary test will generally result in volumetric strain increments together with deviatoric ones. Both dilatant or contractant volumetric strains can be observed, depending on the stress level and void ratio. In most conditions, the grain material itself can be considered as incompressible, so the volume change of the granular medium is due to void ratio changes. So called 'undrained conditions' refer to situations where void ratio changes are excluded, due to unmovable water saturing the sample-a common situation in geomechanics. A proper modelling of dilatancy of granular media allows us to represent both drained and undrained behaviour, and more generally any situation in which coupling between grain skeleton deformation and pore fiuid flow has to be considered. CLoE laws allow a direct formulation of volumetric versus axial strains on basic paths, which are drained axisymmetric triaxial paths.

\subsection{Internal friction}

This is essential in the behaviour of granular materials. As a first approximation, shear strength of a specimen is proportional to the isotropic part of the stress tensor applied. However, this linear dependency on the stress level is only an idealization of the behaviour of real materials, since experimental observations, on axisymmetric triaxial tests for instance, show a continuous evolution of stress and volumetric strain versus axial strain curves when increasing lateral pressure. In the first version presented here, CLoE laws are restricted to the stress level independent idealized material. Hence, all moduli and stress responses will be linear functions of the isotropic pressure, and volumetric strain versus deviatoric strain response will be independent of it.

\subsection{Limit surface}

A main and well known experimental fact about soil behaviour is the existence of a failure or bound or limit surface in stress space. ${ }^{7}$ This surface splits the stress space into two sets: the set of admissible stress states and the set of forbidden stress states. Defined in this way, this surface can only be asymptotically reached. Moreover, we assume that this surface is attainable on triaxial axisymmetric paths. This point is discussed in Section 3.3 .

CLoE laws incorporate explicitly a limit surface; moreover, the constitutive equations are formulated in such a way that no outer stress rate can be generated for a stress state lying on the limit surface (consistency condition).

\subsection{Irreversibility}

Granular materials show strong irreversibility on unloading on a given loading path: this is an illustration of material incremental non-linearity (reverse strain increment does not result in reverse stress increment). More generally, experiments show that any change in the incremental loading direction (in the strain rate, stress rate, or combined space) induces non-proportional material responses: real materials are thoroughly non-linear rather than bi-linear (loading, unloading) or multi linear (multi mechanism plasticity). CLoE laws are basically incrementally thoroughly non-linear, as explained later on. 


\subsection{Cyclic loading}

Reloading after a loading/unloading sequence on a granular specimen will generally produce a response quite different from virgin loading; this is conveniently modelled in the plasticity framework by the concept of an elastic domain inside a so-called loading surface in the stress space, the size of which can be controlled by a hardening law. However, a continued loading. unloading process on a real specimen (cyclic loading) will produce an accumulation of strain, both deviatoric and isotropic. This accumulation will be continuing, or vanishing, depending on loading parameters. Such behaviour is not easily modelled; models accounting for cyclic behaviour have to incorporate discrete memory variables, non-isotropic hardening with backstress, or other rather elaborated features. CLoE v1.00, described here, is not intended to model cyclic behaviour. The response obtained will be ratcheting on some cyclic loading path.

\subsection{Rotation of principal stress directions}

The response of granular materials to principal stress rotation has not been extensively studied up to now. Experimental tests allowing a proper investigation of this response are becoming more common: torsional hollow cylinder tests, ${ }^{8}$ Arthur's DSC, ${ }^{9}$ Grenoble's shear apparatus $1 \gamma 2 \varepsilon^{10}$; a more comprehensive data base will be available in the next years on that important aspect of material behaviour. It is worth noting that principal stress rotation occurs in shear band initiation and development. These phenomena are mainly governed by shear moduli. In CLoE laws, shear moduli are explicitly linked to the localization criterion.

\subsection{Anisotropy}

Anisotropy of granular materials can be significant. The mode of deposition, the particle shape, among other features, can lead to an inherent anisotropy; ${ }^{11}$ moreover, when subjected to a loading process, the material will deform irreversibly and acquire induced anisotropy. ${ }^{12}$ In the present state of its development, CLoE laws apply only to isotropic materials.

\subsection{Strain softening}

This is still a controversial point in experimental characterisation of granular material behaviour. Non-homogeneities in the deformation field in rheological tests have been recognized by different experimental works; in axisymetric triaxial tests, X-ray techniques ${ }^{13}, 14$ and more recently tomodensitometry (CT $\operatorname{scan}^{13-17}$ ) have shown that rigid cones are induced by the friction of the ends of the specimen on the plattens and that lubrication and reduced slenderness of the specimen can improve the homogeneity. Improved tests show less pronounced peaks in the stress-strain curves, which induces the idea that experimentally observed softening responses can be an effect of strain localization, or other diffuse heterogeneous deformation mode (bulging, ...). On the other hand, one can still consider that at least a part of the observed softening is a rheological phenomenon, due to some kind of damaging process in the granular structure (for example, void ratio inflation in dilatant materials ${ }^{18}$ ). The authors of the present paper do not reject the second hypothesis, however, CLoE laws have been developed on the basis of non* softening elementary behaviour, as a first step.

\subsection{Strain localization}

This is observed as the final mode of deformation in most of the elementary tests performed on granular materials when rupture is approached. Theoretical works by $\mathrm{Hill}_{,}{ }^{19} \mathrm{Mandel}^{20}{ }^{20} \mathrm{Rice},{ }^{21}$ Vardoulakis $^{22}$ and others have shown that, when considered as a bifurcation phenomenon, strain 
localization can be predicted on the basis of a so-called localization criterion. The criterion involves essentially the constitutive equation of the material. As discussed in detail in Reference 6, serious difficulties arise when deriving the criterion for non-linear incremental constitutive equations. Desrues and Chambon have shown in Reference 23, a heuristic example of a general criterion obtained without linearization for a specific form of incremental non-linear constitutive equations. Loret presented in Reference 24, further developments in the same spirit. CLoE laws are based on a mathematical form which allows us to derive a general criterion for strain localization along the same lines; it is one of their essential features, which will be discussed in a following paper.

\section{THEORETICAL BASIS}

\subsection{Rate type constitutive equations: the basic concepts}

Despite the conceptual differences between CLoE models and elastoplastic constitutive equations, both belong to the rate-type non-linear models framework. Kolymbas ${ }^{25}$ has proposed to call hypoplastic, those non-linear models which are not based on flow theory of plasticity. In this section we discuss a number of general requirements which have to be met for any model in this framework.

Due to irreversibility of the material behaviour of granular materials, any direct stress--strain formulation is ruled out. However, irreversibility can be conveniently captured in a rate-type theory. In such a theory, stress rate (an objective one) and strain rate are supposed to be related by a tensorial function, depending on some state variables. The tensorial function can be linear, piecewise linear or non-linear. Linearity reduces the application to reversible materials (hypoelasticity); so it is of little interest for granular materials. Piecewise linearity is obtained when the tensorial function is built up by some procedure introducing a partition in the stress rate space, for example, including one or several loading/unloading criterions: the simplest form of flow theory involves two linear zones, depending on the direction of the stress rate with respect to a loading surface. More elaborate elastoplastic constitutive equations may involve four, eight, potentially a non-limited number of linear zones, depending on the number of plastic potentials considered. Thoroughly non-linear constitutive equations are the generalization of piecewise linear ones, when linear cones reduce to a vanishing cone on any given direction. ${ }^{21,26}$

A general form for rate-type constitutive equations is

$$
\stackrel{\nabla}{\sigma}=\underline{f}(\underline{\sigma}, V, \underline{\dot{\varepsilon}})
$$

with $\stackrel{\nabla}{\sigma}$ an objective stress rate, $\underline{\sigma}$ the stress state, $\underline{\dot{\varepsilon}}$ the strain rate, and $V$ a set of state variables. The evolution law for the state variables has to be specified in the constitutive model.

For a non-viscous material, $f$ is only required to be a tensor valued function of the first degree with respect to the strain rate, depending on the state and the history (described by a finite set of internal variables) of the material. It is necessary that, given the history, the equation gives a unique stress rate response to any strain rate; the converse is not required.

The form

$$
\underline{\dot{\varepsilon}}=\underline{f}^{-1}(\stackrel{\nabla}{\sigma}, \underline{\sigma}, V)
$$

is often used. It is suitable for classical elastoplasticity as the strain rate has to be divided in elastic 
and plastic parts. It is also sometimes used in endochronic models ${ }^{27}$ and in incremental constitutive equations. ${ }^{28}$ In that form it is necessary to check if $f^{-1}$ is a one-to-one correspondence, which is not an easy task if the law is somewhat complicated. Unfortunately, for some incremental laws $f^{-1}$ has turned out not to meet this condition. ${ }^{3,4}$

\subsection{Incremental continuity}

If the function $f$ is discontinuous, then serious problems will occur in numerical computation as the same problem run on two different computers may lead to distinct results. ${ }^{29}$

Gudehus ${ }^{30}$ performed a comparison between a number of constitutive equations for soils. He proposed to summarize the properties of the function $f$ by a graphical representation (Figure 1). This representation can be understood as a mapping between the strain rate space and the stress rate space: as the stress rate is an homogeneous function of first degree with respect to the strain rate, the stress rate-strain rate relation is fully described by the mapping between an hypersphere of unit radius centered at the origin of the strain rate space and the corresponding hypersurface in the stress rate space. This representation is very useful and we are using it extensively in the sequel. However, the two-dimensional Gudehus diagram shows only a part of the problem as strain and stress rate spaces are six dimensional. Constitutive equations which look continuous in that diagram may be actually discontinuous.

It is sometimes rather difficult to meet the continuity condition for incremental interpolated laws and this has led to somewhat complicated formulations in previous attempts to overcome the problem: References 3, 31 and 4.

\subsection{Limit surface}

Experimental evidence of the existence of a limit surface in the stress space, discussed before, has a few important consequences on the theoretical foundation of constitutive equations. It implies that:

(1) the Cauchy stress is a member of the set of state variables;

(2) we must have got a limit surface which is an hypersurface in the stress space which define two parts: the inner part plus the limit surface is the set of admissible stress states; stress states in the outer part are not physically admissible.
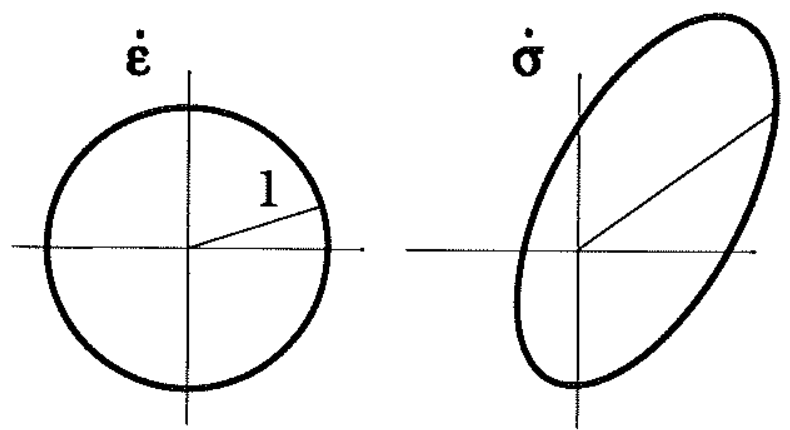

Figure 1. Gudehus graphical representation: the right hand figure is the mapping of the stress rate responses to the set of unit strain rate vectors represented as a circle on the left. 
So in a constitutive equation we have to distinguish between physically admissible and unadmissible stress states. When no explicit limit surface is incorporated in a model, it needs to be implicitly included; otherwise the model would not meet an essential feature of soil behaviour.

Moreover, experimental results (especially classical triaxial tests) clearly show that for some stress states, vanishing stress rates can be observed for peculiar non-vanishing strain rates. The material will flow under constant stress if and only if the strain rate is coincident with that peculiar strain rate direction. This kinematical condition for flow is usually called the flow rule. There is no mathematical reason to assume that the limit surface (in the sense defined before) can be experimentally reached in classical triaxial tests and thus coincide with the set of stress states, for which a large strain increment is possible for steady states of stress. However, this assumption is commonly made in numerous classical elastoplasticity soil models. If this assumption is discarded, a kind of strain softening arises necessarily in the constitutive equation. This means that the constitutive equation is no more invertible. As we want that CLoE does not exhibit any strain softening, we assume that upon the limit surface, a flow is possible without any stress variation. According to our assumption, when the stress is lying on the limit surface, a strain rate direction exists in which an arbitrary large strain rate increment can be applied without inducing any stress increment. So regarding the constitutive tensorial function $f$, the limit surface is characterised by the fact that $f$ is no more invertible. For any other directions-i.e. not meeting the flow rule - the stress rate must be directed inwards. If this condition is not met, then for some loading paths the stress path will cross the limit surface, which is not admissible. Figure 2 is a graphical representation of these properties using the Gudehus symbolic diagram. We assume that the limit surface has a tangential plane (we will select surfaces having this property everywhere). Then it appears as an hyperplane in the stress rate space, including the space origin. The right figure corresponds to an inconsistent equation, because for some strain rates the corresponding stress rates are directed outwards with respect to the limit surface. On the left figure, corresponding to a consistent equation, the stress rate envelope is tangent to the limit hyperplane; for any strain rate, the stress rate can be either null (only one strain rate gives that response), or finite but directed inwards.

Let the limit surface equation be

$$
\psi(\sigma)=0
$$

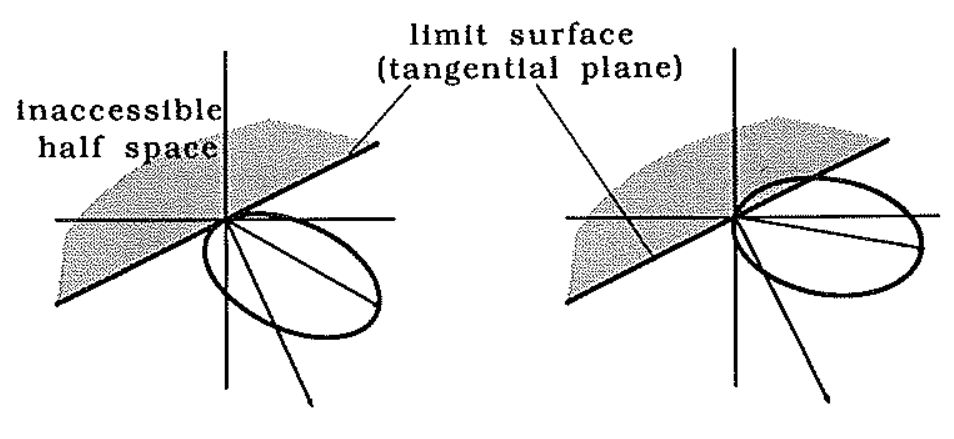

(a) consistent response

(b) Inconsistent response

Figure 2. Consistency with respect to limit surface: the response envelop for a state lying on the limit surface can be tangent to the surface like in (a) but not secant like in (b). 
the inner part is characterized by

$$
\psi(\sigma)<0
$$

and the limit surface consistency condition reads

$$
\frac{\partial \psi}{\partial \sigma_{i j}} \stackrel{\nabla}{\sigma} \sigma_{i j} \leqslant 0
$$

which expresses that the scalar product of the stress rate by the outer normal to the limit surface has to be negative or null since the stress rate is null or directed inwards.

The only assumption made to derive this condition is that the limit surface is only characterized by the stress state (and not by the other state variables). It is, however, possible to define other critical surfaces depending on other state variables. For these cases one may deduce other consistency conditions. This is not in the scope of the present discussion.

\section{THE NEW CLASS OF CONSTITUTIVE EQUATIONS}

The description of the main features of CLoE will follow a Top-Down analysis. We will start from the most general formulation of a rate type constitutive law, and introduce step by step the special choices made. At every step, the theoretical objects should be reduced to lower-level simplest objects; eventually, the bottom level will be reached when all the theoretical objects introduced in previous steps have been reduced to experimentally identifiable objects.

\subsection{Guidelines for a conceptual comparison of CLoE versus elastoplastic constitutive equations}

Table I gives in a synoptic form a few comparison points between elastoplastic type constitutive equations and CLOE. The first line indicates that in CLoE, no separation is assumed into plastic and elastic parts of the strain rate; the general form of the stress- strain rate equation on line 2 shows that CLoE does not use the concept of plastic potential $g$ and elastic compliance $\underline{\mathbf{D}}^{-1}$ but a set of two constitutive tensors $\mathbf{A}$ and $\mathbf{b}$ depending on state variables.

As far as geometric entities defined in principal stress space are concerned, CLoE knows only a limit surface which is equivalent to the bounding surface of elastoplasticity; nothing like a yield

\begin{tabular}{|c|c|c|}
\hline & elastoplasticity & CLoE \\
\hline $\begin{array}{l}\text { Strain rate decomposition into } \\
\text { elastic and plastic parts }\end{array}$ & $\underline{\dot{\varepsilon}}=\underline{\dot{\varepsilon}}^{e}+\underline{\dot{\varepsilon}}^{p}$ & $\dot{\varepsilon}$ global \\
\hline $\begin{array}{l}\text { Stress strain } \\
\text { Rate equation }\end{array}$ & $\underline{\dot{\varepsilon}}=\underline{\underline{\mathbf{D}}}^{-1} \underline{g}+\lambda \partial g / \partial \underline{\sigma}$ & $\underline{\underline{\sigma}}=\underline{\underline{\mathbf{A}}}: \underline{\underline{\varepsilon}}+\underline{\mathbf{b}}\|\underline{\dot{\varepsilon}}\|$ \\
\hline Stress space geometrical entities & $\begin{array}{l}\text { Yield surface } f(\sigma)=0 \\
\text { plastic potential } g(\sigma)=0 \\
\text { bounding surface }\end{array}$ & $\begin{array}{l}\text { Limit surface and consistency } \\
\text { at LS and flow rule }\end{array}$ \\
\hline Hardening law & Yield surface evolution law & Basic paths formulation \\
\hline Dilatancy. & Standard versus non-standard & Basic paths formulation \\
\hline Interpolation & Yield surface + plastic potential & $\begin{array}{l}\text { Interpolation on Lode's angle } \\
\text { between axisym states }\end{array}$ \\
\hline
\end{tabular}

Table I. Guidelines for conceptual comparison of CLoE laws with elastoplastic laws 
surface is defined. However, when reaching the limit surface, a consistency equation has to be met.*

The hardening law is more or less an elastoplastic concept, based on the idea of an elastic domain increasing in size as plastic strain progresses; however, the hardening law and the corresponding evolution equation for plastic potential play an essential role even in monotonic loading, as they control the tangent stiffness of the model through the plastic multiplier(s). In CLoE laws, the stress strain response on a set of basic paths is directly formulated; no hardening law needs to be superimposed.

The same applies to the modelling of volume changes: in elastoplastic models, introduction of so-called non-standard plastic potentials has been necessary to correct the unrealistic volumetric plastic strain predicted when using yield surfaces as plastic potentials (standard plastic procedure). Alternatively, in Cam clay models, a yield surface has been identified to a physically admissible plastic potential, with regard to volumetric plastic strains. In CLoE laws, volumetric strain versus deviatoric strain response is directly formulated together with stress-strain response on the basic paths mentioned before.

The last line of the table concerns interpolation. For any type of incremental constitutive equations, generality requires that a response be defined whatever the stress state is inside the limit surface. In the elastoplastic framework, generalization of constitutive equations can be seen at first as 'natural' because it is controlled by the form of the yield surfaces and plastic potentials; however, available experimental information is restricted to a limited number of stress-strain paths; hence the form of the yield surface and plastic potential is by no way an experimental evidence, but a matter of modeller's choice. The same applies to CLoE laws, in which an interpolation procedure between axisymetric paths is explicitly built-up (described in Section 4.9).

\subsection{Generic mathematical form}

A main feature of soil behaviour is irreversibility, which leads to a non-linear constitutive tensor function $f$.

In classical elastoplasticity, incremental irreversibility is obtained through one or a few loading criterions (which is a serious drawback for a bifurcation analysis). CLoE laws have a unique expression for the tensor function, which has the simple form

$$
\underline{\nabla}=\underline{\underline{\mathbf{A}}}: \underline{\dot{\varepsilon}}+\underline{\mathbf{b}}\|\underline{\dot{\varepsilon}}\|
$$

in which $\underline{\underline{A}}$ is a four rank tensor and $\underline{\mathbf{b}}$, a second rank one. $\underline{\underline{\mathbf{A}}}$ and $\underline{\mathbf{b}}$ depend on state variables.

It can be seen that, given a state, $\underline{\mathbf{A}}$ and $\underline{\mathbf{b}}$ are fixed and $\underline{\mathbf{b}}$ controls the difference between responses to $\underline{\dot{\varepsilon}}$ and $-\underline{\dot{\varepsilon}}$. The incremental non-linearity is due to the norm $\|\dot{\varepsilon}\|$.

Another useful equivalent form is

$$
\stackrel{\underline{\sigma}}{\underline{\sigma}}=\underline{\underline{\mathbf{A}}}:\left(\underline{\dot{\varepsilon}}+\underline{\mathbf{b}^{\prime}}\|\underline{\dot{\varepsilon}}\|\right)
$$

The general form has been chosen in the perspective of non-linear shear band analysis. It defines a class of models which is an extension of a heuristic model proposed in References 32 and 23. In these references, the aim was to emphasize the need of a true non-linear shear band analysis

\footnotetext{
* in this context, consistency equation has the same meaning as in perfectly plastic laws.
} 
when non-linear models are considered. In the present development the non-linear band analysis is conserved while the model structure is elaborated to encompass real geomaterial behaviour. The shear band analysis for CLoE is the subject of a forthcoming paper.

Similar formulations have been proposed by Kolymbas ${ }^{33}$, by Lanier for its simplified model ${ }^{34}$ and in endochronic theory by Valanis. ${ }^{35}$ But, contrary to some previous works, we do not intend to have an explicit a priori formula for $\underline{\underline{\mathbf{A}}}$ and $\underline{\mathbf{b}}$ as functions of the state variables. $\underline{\underline{\mathbf{A}}}$ and $\underline{b}$ components will be obtained on the basis of an interpolation procedure between $\underline{\underline{\mathbf{A}}}$ and b determined on special stress paths-the well known axisymetric stress paths.

This approach is not contradictory with the ones based on representation theorems of isotropic tensor valued functions of tensorial arguments. ${ }^{25,34}$ The accent here is put on the way $\underline{\underline{A}}$ and $\underline{\mathbf{b}}$ are linked to experimentally accessible stress strain paths.

\subsection{State variables}

When considering soils as continuum media, some state variables have to be defined to reflect at the macroscopic level the characteristics of the grain assembly, such as distribution of contacts and contact forces. Stress state void ratio, and more complex variables like fabric tensors can be incorporated in the set of state variables.

In elastoplastic constitutive equations, stress state is always considered as a state variable; in addition, other variables have to be defined to control the evolution of the load surface(s) and plastic potential(s). If kinematic hardening is considered, a tensorial state variable is taken into account, in addition to stress state. With multi-surface models, the number of state variables can be rather large.

$C L O E v 1.00$ has been made as simple as possible: The Cauchy stress tensor is the only state variable considered. Some limitations of the model result from this choice; as mentioned in section 2, cyclic behaviour will not be properly modelled with this version.

The previous assumption leads to a few important consequences. Using theories of isotropic tensor value functions depending on tensorial variables, as the constitutive function $f$ depends only on one second rank tensor - the stress tensor - Chambon [3] has established the following results:

- the constitutive equations are cross-anisotropic in the principal axes of the stress.

Then, using the classical representation of symmetric 2-rank tensors as 6-components vectors, we can write

$$
\underline{x}=\left\{x_{11}, x_{22}, x_{33}, x_{23}, x_{31}, x_{12}\right\}^{T}
$$

and the tensor of moduli $\underline{\underline{\mathbf{A}}}$ takes the form:

$$
\underline{\underline{\mathbf{A}}}=\left[\begin{array}{llllll}
a & f^{\prime} & e^{\prime} & & & \\
f & b & d^{\prime} & & & \\
e & d & c & & & \\
& & & g & & \\
& & & & h & \\
& & & & & j
\end{array}\right]
$$

when expressed in principal axes of the stress state. This figure is typical of cross-anisotropy. In the same axes, $\underline{b}^{3}$ reads

$$
\underline{\mathbf{b}^{\prime}}=\{k, l, m, 0,0,0\}^{\mathrm{T}}
$$


if two principal stresses are equal-as in a triaxial axisymmetric state-the function $f$ is tranversely isotropic with the same revolution axis as the stress tensor.

For stress states lying on the axis $\sigma_{1}, \sigma_{2}$ or $\sigma_{3}$ in the deviatoric plane, the constitutive tensors $\underline{\underline{\mathbf{A}}}$ and $\underline{\mathbf{b}}^{\prime}$ have the simpler form, involving only eight independent moduli $\left\{a, b, d, e, e^{\prime}, j, k, l\right\}$ :

$$
\underline{\underline{\mathbf{A}}}=\left[\begin{array}{llllll}
a & e^{\prime} & e^{\prime} & & \\
e & b & d & & \\
e & d & b & & \\
& & & b-d & \\
& & & j & j
\end{array}\right]
$$

if the three principal stresses are equal, the state is isotropic, and the function $f$ is also isotropic. The tensor of moduli $\underline{\underline{A}}$ takes the form

$$
\underline{\underline{\mathbf{A}}}=\left[\begin{array}{llllll}
a & d & d & & & \\
d & a & d & & & \\
d & d & a & & & \\
& & & a-d & & \\
& & & a-d & \\
& & & & a-d
\end{array}\right]
$$

when expressed in the principal axes of the stress state. In the same axes, b' reads

$$
\underline{\mathbf{b}^{\prime}}=\{k, k, k, 0,0,0\}^{\mathrm{T}}
$$

\subsection{A fundamental property: flow rule}

Given the mathematical form of the stress-strain rate relation, and the equation of the limit surface, a fundamental property of the constitutive equations can be derived, which can be identified as a flow rule in the spirit discussed in Section 3.3. This property will be used in the sequel, namely when deriving the equation for consistency at the limit surface and in the bifurcation analysis.

We assume in the following that the first constitutive tensor $\mathbf{A}$ can be inverted. This cannot be proven in general, as it depends on the way $\underline{\underline{A}}$ is built-up in the constitutive equations. The assumption is credible if moduli computation and interpolation are based on independent stress paths.

Let $\underline{\bar{\varepsilon}}$ be a normalized strain rate:

$$
\|\underline{\dot{\varepsilon}}\|=1
$$

Using the general form of the law and the inversibility of $\underline{\underline{\mathbf{A}}}$,

$$
\begin{gathered}
\underline{\dot{\bar{\varepsilon}}}=\underline{\underline{\mathbf{A}}}^{-1} \underline{\underline{\nabla}}-\underline{\mathbf{b}}^{\prime} \\
\|\underline{\dot{\varepsilon}}\|^{2}=\left(\mathbf{A}_{i j k l}^{-\frac{1}{\sigma}} \stackrel{\nabla}{\sigma}_{k l}-\mathbf{b}_{i j}^{\prime}\right)\left(\mathbf{A}_{i j m n}^{-1} \stackrel{\nabla}{\sigma_{m n}}-\mathbf{b}_{i j}^{\prime}\right)=1
\end{gathered}
$$


or

$$
\mathbf{A}_{i j k l}^{-1} \mathbf{A}_{i j m n}^{-1} \stackrel{\nabla}{\sigma_{k l}} \underset{\nabla}{\sigma_{m n}}-2 \mathbf{b}_{i j}^{\prime} \mathbf{A}_{i j k l}^{-1} \stackrel{\nabla}{\sigma_{k l}}+\left\|\mathbf{b}^{\prime}\right\|=1
$$

Separating the norm $\mu$ and the direction $\underline{\dot{\Sigma}}$ of the stress rate tensor $\underline{\nabla}$ :

$$
\stackrel{\nabla}{\sigma}=\mu \underline{\dot{\Sigma}}
$$

with $\|\underline{\dot{\Sigma}}\|=1$, one obtains a second-order equation in $\mu$,

$$
\mu^{2} \mathbf{A}_{i j k l}^{-1} \mathbf{A}_{i j m n}^{-1} \dot{\Sigma}_{k l} \dot{\Sigma}_{m n}-2 \mu \mathbf{b}_{i j}^{\prime} \mathbf{A}_{i j k l}^{-1} \dot{\Sigma}_{k l}+\left\|\mathbf{b}^{\prime}\right\|=1
$$

The solutions of this equation express the stress rates corresponding to a unit strain rate, given the stress rate direction $\underline{\dot{\Sigma}}$. The equation has generally two roots in $\mu$. Remembering the Gudehus diagram (Figure 1) it is clear that:

(1) for any stress state one root is positive and the second is negative (origin of the axes is inside the diagram),

(2) for a stress state on the limit surface one root is null (origin of the axes is lying on the diagram).

So the product of the two roots has to be negative or null. The product is equal to the ratio $c / a$ of second- and zero-order coefficients. As the first coefficient $a$ is squared,

$$
a=\mathbf{A}_{i j k i}^{-1} \mathbf{A}_{i j m n}^{-1} \dot{\Sigma}_{k l} \dot{\Sigma}_{m n}
$$

is positive. So the third coefficient

$$
c=\left\|\mathbf{b}^{\prime}\right\|-1
$$

has to be negative or null; this leads to

$$
\left\|\mathbf{b}^{\prime}\right\|<1
$$

or

$$
\left\|\mathbf{b}^{\prime}\right\|=1
$$

when the stress state is lying on the limit surface.

This last equation can be obtained by an other way. At the limit surface one strain rate gives a null stress rate

$$
\underline{\dot{\sigma}}=\underline{\underline{\mathbf{A}}}\left(\underline{\dot{\varepsilon}}+\underline{\mathbf{b}}^{\prime}\|\underline{\dot{\varepsilon}}\|\right)=0
$$

As $\underline{\underline{\mathbf{A}}}$ is invertible, we obtain

$$
\underline{\mathbf{b}^{\prime}}=\frac{\underline{\dot{\varepsilon}}}{\| \underline{\dot{\varepsilon} \|}}
$$

This is the flow rule of our constitutive equation, as it has been defined in Section 3.3.

It implies

$$
\left\|\underline{\mathbf{b}^{\prime}}\right\|=1
$$




\subsection{Consistency at the limit surface}

When the stress state reaches the limit surface, consistency requires that no outer stress rate response can be generated by the constitutive equations.

As previously discussed in equation (5), in Section 3.3 , this condition reads as

$$
\frac{\partial \psi}{\partial \sigma_{k l}} \stackrel{\nabla}{\sigma l}_{k l}<0
$$

In this form, the consistency condition limits the stress increment but does not give a useful condition on the constitutive tensors $\mathbf{A}$ and $\underline{\mathbf{b}}$. In order to obtain such a condition, the development of the preceeding section will be pursued.

Observing that at the limit surface

$$
\left\|\underline{\mathbf{b}^{\prime}}\right\|=1
$$

one obtains a new form of (16) as

$$
\mu^{2} \mathbf{A}_{i j k l}^{-1} \mathbf{A}_{i j m n}^{-1} \dot{\Sigma}_{k l} \dot{\Sigma}_{m n}-2 \mu \mathbf{b}_{i j}^{\prime} \mathbf{A}_{i j k l}^{-1} \dot{\Sigma}_{k l}=0
$$

the first term is square, so for the second we have,

$$
-2 \mu \mathbf{b}_{i j}^{\prime} \mathbf{A}_{i j k l}^{-1} \dot{\Sigma}_{k l}<0
$$

This means that $\underline{\dot{\Sigma}}$ belongs to a half-space, which has to coincide with the inner side of the limit surface. Rewriting the consistency equation (22) as follows:

$$
\mu \frac{\partial \psi}{\partial \sigma_{k l}} \dot{\Sigma}_{k l}<0
$$

allows us to remark that both (26) and (25) have the same structure, and have to be true whatever $\dot{\Sigma}_{k l}$ is; it results that

$$
-2 \mu \mathbf{b}_{i j}^{\prime} \mathbf{A}_{i j k l}^{-1}=\lambda \mu \frac{\partial \psi}{\partial \sigma_{k l}}
$$

with $\lambda>0$.

This result can be rearranged as

$$
\frac{\partial \psi}{\partial \sigma_{k l}}=-\lambda \mathbf{A}_{i j k l}^{-1} \mathbf{b}_{i j}^{\prime}
$$

or

$$
\mathbf{A}_{k i t j}^{-1} \frac{\partial \psi}{\partial \sigma_{k l}}=-\lambda \mathbf{b}_{i j}^{\prime} \text { with } \lambda>0
$$

This equation is a new form of the consistency equation (22), which will be prefered in the sequel as it involves explicitly the constitutive tensors $\underline{\underline{\mathbf{A}}}$ and $\underline{\mathbf{b}}^{\prime}$.

\subsection{Limit surface equation}

The equation of the limit surface is only dependent on the stress state

$$
\psi(\sigma)=0
$$


Let us notice that if $\mathbf{b}^{\prime}$ is expressed as a function of $\sigma$, then equation (21) is equivalent to equation (29).

As far as isotropic materials are concerned, the orientation of principal stress direction with respect to material ones is not significant. Then the stress state is resumed by a set of three variables, namely the principal stresses or any other set of three independent invariants. We are using

$I_{\sigma}=J_{1}=\sigma_{1}+\sigma_{2}+\sigma_{3}$ the first invariant of stress;

$I I_{\sigma}^{2}=\left(\sigma_{1}-\sigma_{2}\right)^{2}+\left(\sigma_{2}-\sigma_{3}\right)^{2}+\left(\sigma_{3}-\sigma_{1}\right)^{2}$ the second invariant;

$\cos (3 \phi)$ the phase angle in the deviatoric plane.

It is well known that the limit friction angle is independent of compression and extension triaxial stress paths, and that limit surfaces have to be regular and derivable. Compared to other proposed forms of limit surface equations (for example Lade, ${ }^{36}$ Matsuoka-Nakai, ${ }^{37}$ basic Mohr-Coulomb's surface, Drucker-Prager), the Van Eekelen surface ${ }^{38}$ meets these two requirements. It reads

$$
\psi=\frac{I_{\sigma}}{I_{\sigma}}-f\left(I_{\sigma}\right) \alpha(1-\beta \cos 3 \phi)^{n}
$$

where $f\left(I_{\sigma}\right)$ is a function of the mean stress, which accounts for mean stress-level dependency; in CLoEv1 $00, f\left(I_{\sigma}\right)=1$ is considered, $n$ is a material parameter controlling the shape of the surface. $\alpha, \beta$ are material parameters determined by axisymmetric triaxial compression and extension tests.

In terms of stress invariants, $\psi(\sigma)$ takes the form

$$
F\left(I_{\sigma}\right)=a I_{\sigma}\left(1+b 27 \sqrt{2} \frac{I I_{\hat{\sigma}}}{I I_{\hat{\sigma}}^{3}}\right)^{n}-I_{\hat{\sigma}}
$$

with $\hat{\sigma}$ the stress deviator and $I I_{\hat{\sigma}}=1 / 3 \operatorname{tr}\left(\hat{\sigma}^{3}\right)$.

This form is more convenient for derivation with respect to stress components.

Figure 3 illustrates the section of van Eekelen surface in the deviatoric plane for $\phi_{c}=45^{\circ}$, $\phi_{e}=46^{\circ}, n=-0,2$.

\subsection{A Complementary hypothesis: stress level dependency}

Following the discussion in the paragraph in Section 2.3, a complementary hypothesis is introduced: the material response is supposed to be simply linear dependent on the stress level.

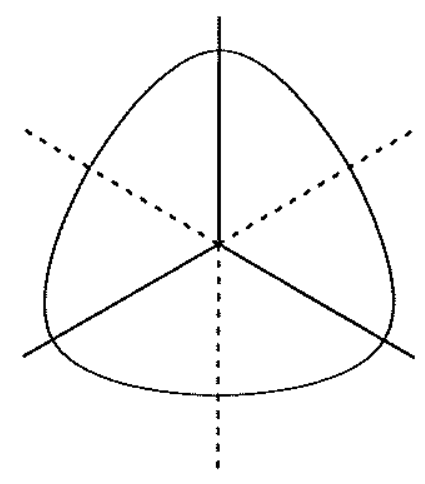

Figure 3. Van Eekelen limit surface in the deviatoric plane. 
Clearly this is only a first order approximation of the actual behaviour of geomaterials, however, it is admissible for moderate variations of the stress level. With this assumption, the stress ratio versus axial strain and volumetric versus axial strain curves, being non-dimensional, are unique for a given material whatever the mean stress is; and the constitutive tensors $\underline{\underline{A}}$ and $\underline{b}^{\prime}$ are simply linearly dependent on the trace of the stress tensor.

\subsection{Axisymmetric image stress states and image laws}

One basic feature of the $C L O E$ class of models is given a stress state, $\underline{A}$ and $\mathbf{b}^{\prime}$ are determined by an interpolation procedure between the responses for axisymmetric triaxial states.

We define two images of the actual stress point in the three-dimensional stress space (principal stresses) according to Figure 4.

These image states lie on the axisymmetric triaxial axes in the deviatoric plane of the actual state, and their deviatoric stress versus limit deviatoric stress ratio $\bar{q}$ is the same as that for the actual state. Lode's angle, will be the basic co-ordinate for the subsequent interpolation.

As discussed in Section 4.3, constitutive tensors $\underline{\underline{\mathbf{A}}}$ and $\underline{\mathbf{b}^{\prime}}$ have a simpler form for stress states lying on the axis $\sigma_{1}, \sigma_{2}$ or $\sigma_{3}$ in the deviatoric plane:

$$
\begin{aligned}
\underline{\mathbf{A}}= & {\left[\begin{array}{llllll}
a & e^{\prime} & e^{\prime} & & & \\
e & b & d & & & \\
e & d & b & & \\
& & & b-d & & \\
& & & & j & \\
& & & & j
\end{array}\right] } \\
\underline{\mathbf{b}^{\prime}}=\{k, l, l, 0,0,0\}^{\mathrm{T}} &
\end{aligned}
$$

Their expression involves only eight unknown quantities.

The axisymmetric triaxial test is the most popular test in soil mechanics; relevant data are easy to obtain for the material response on axisymmetric stress paths, either by performing an actual testing program on the given material or by consulting existing soil data bases.
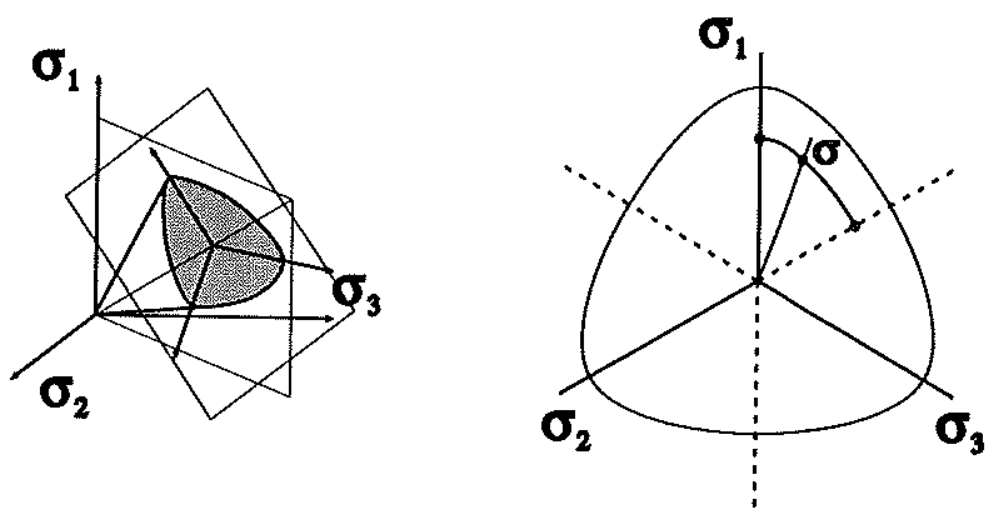

Figure 4. image states in the deviatoric plane: two axisymmetric stress states (compression and extension) defined by projection of the actual stress state on the axisymmetric axis of the plane. 


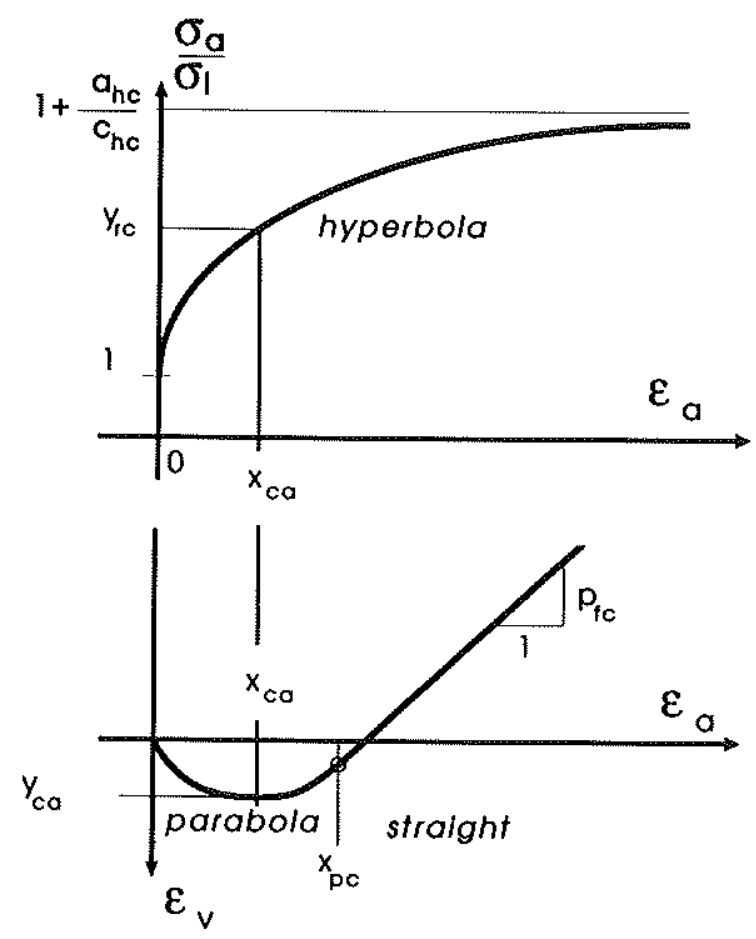

Figure 5. Triaxial compression response. The basic paths are defined by analytical formulation: here is shown the formulation of the axisymmetric triaxial compression response. The quantities $y_{r}, x_{c a}$, etc, are determined for a given material by an identification procedure which takes as input a set of easily accessible geotechnical parameters.

The determination of the unknown quantities ( $j$ excepted) can be made on the basis of analytical formulations ${ }^{\dagger}$ of the axisymmetric triaxial responses in compression and in extension (loading) (Figures 5 and 6) plus some additional information from incipient unloading responses, and so-called 'pseudoisotropic' response (Figure 7). All these stress paths are easy to realize on conventional triaxial apparatus.

The analytical responses have to ensure the consistency to the limit surface, which is easy to do for axisymmetrical paths - the asymptote of the load curve has to match the friction angle introduced in the limit surface-but needs a discussion for a pseudoisotropic response (not detailed here).

Moreover, at an isotropic stress state some of the analytical paths are redundant. This implies some links between the slopes of the analytical curves at the isotropic point, which in turn results in constraints to deal with in the identification process (Section 5.1).

The case of the shear modulus $j$ is special; it must be discussed in relation with bifurcation analysis, see References 39 and 40 . This is another basic feature of CLoE.

\subsection{Interpolation}

As discussed in Section 4.8, the constitutive response for axisymmetric states is known. So we can easily get the so-called 'image-laws' associated with the 'image states'. We still have to define

\footnotetext{
'more detail on the basic paths formulation can be found in References 39 and 40.
} 


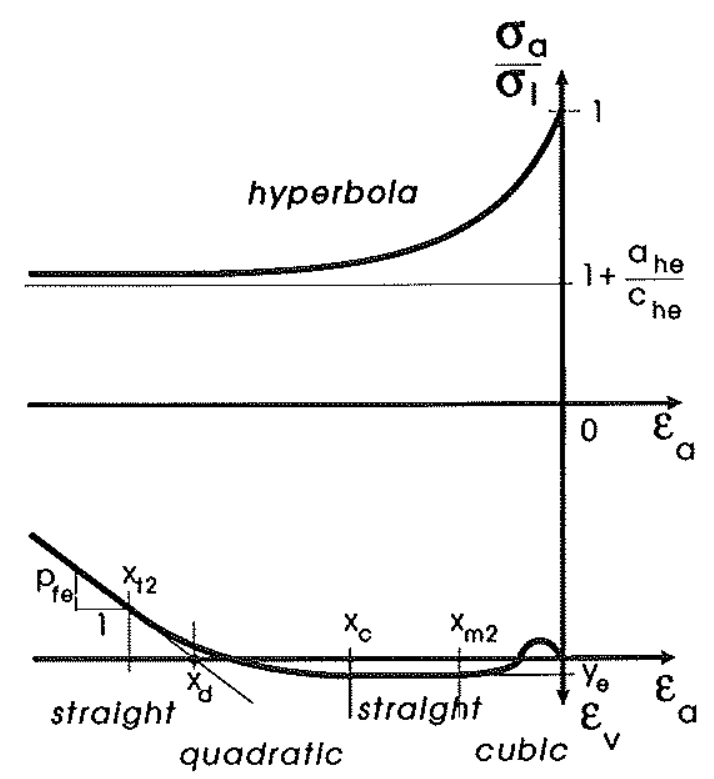

Figure 6. Triaxial extension response: analytical formulation of the response.

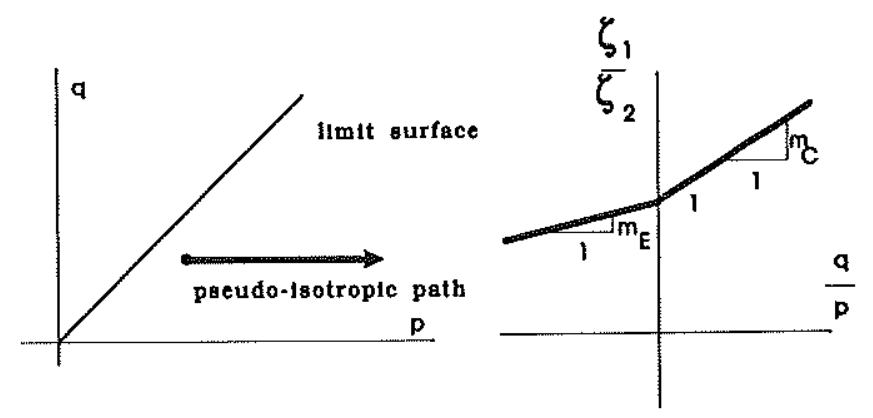

Figure 7. Pseudoisotropic stress path: definition of the path (left). On the right side is represented the assumed characteristic of the ratio of the axial versus radial strain rate as a function of the normalized deviatoric stress.

the response whatever the state is. This is the subject of the next paragraph. The role of the interpolation procedure is to give the actual law (i.e. the $\mathbf{A}$ and $\underline{\mathbf{b}}^{\prime}$ tensors for the actual state) as a function of the image laws and the Lode's angle of the actual stress state.

Some essential features are now pointed out:

(1) $\mathbf{A}$ and $\underline{\mathbf{b}}^{\prime}$ are interpolated separately,

(2) the interpolation procedure must degenerate consistently when the stress state tends towards a triaxial axisymmetric state,

(3) for axisymmetric states consistency is a priori met by the choice of the basic path formulation,

(4) the interpolation scheme ensures consistency at the limit surface by enforcing interpolated $\mathbf{A}$ and $\mathbf{b}^{\prime}$ to meet the consistency equation (28),

(5) the interpolation must be as simple as possible; linear interpolation is the simplest one can 
find. Let us define the interpolation coefficients $\theta^{\mathrm{E}}$ and $\theta^{\mathrm{C}}$ for extension and compression image law tensors as

$$
\begin{gathered}
\theta^{\mathrm{E}}=\frac{\left|\phi^{\mathrm{R}}-\phi^{\mathrm{C}}\right|}{\pi / 6} \\
\theta^{\mathrm{C}}=\frac{\left|\phi^{\mathrm{R}}-\phi^{\mathrm{E}}\right|}{\pi / 6}=1-\theta^{\mathrm{E}}
\end{gathered}
$$

with $\phi^{\mathrm{R}}, \phi^{\mathrm{E}}, \phi^{\mathrm{C}}$ the Lode angle of the actual, image-extension and image-compression stress state (réel, extension, compression).

\subsubsection{Interpolation of ${\underline{\mathbf{b}^{\prime}}}^{\prime}$}

(1) a first interpolation defines the amplitude $a$ of the tensor $\underline{b}^{\text {R }}$,

$$
a=\theta^{\mathrm{E}}\left\|\underline{\mathbf{b}}^{\mathrm{E}}\right\|+\theta^{\mathrm{C}}\left\|\underline{\mathbf{b}}^{\prime \mathbf{C}}\right\|
$$

(2) then an interpolated direction is defined by $\underline{\mathbf{d}}$ as

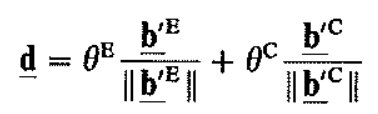

(3) finally the tensor ${\underline{b^{\prime}}}^{\mathbf{R}}$ is defined as having the amplitude $a$ and the same direction as $\underline{d}$. It reads

$$
{\underline{\mathbf{b}^{\prime}}}^{\mathrm{R}}=a \frac{\underline{\mathrm{d}}}{\|\underline{\mathrm{d}}\|}
$$

4.9.2. Interpolation of $\mathbf{A}$ This interpolation has to be done under the constraint of the consistency condition, to be asymptotically met when approaching the limit surface; this constraint leads to the following procedure:

(1) an interpolation on the Lode's angle is done for the actual stress state, giving a first tensor $\underline{\underline{A}}^{\mathrm{R} 1}$ for the actual stress state.

$$
\underline{\underline{\mathbf{A}}}^{\mathrm{R} 1}=\theta^{\mathrm{E}} \underline{\underline{\mathbf{A}}}^{\mathrm{E}}+\theta^{\mathrm{C}} \underline{\underline{\mathbf{A}}}^{\mathrm{C}}
$$

(2) following the same lines for actual states, Image laws at the limit surface (SL $\left.{ }^{t}\right) \underline{A}_{S L}^{\mathrm{E}}, \underline{\mathbf{b}}_{\mathrm{SL}}^{\mathrm{B}}$ and $\underline{\underline{A}}_{S L}^{\mathrm{C}}, \underline{\mathbf{b}}_{\mathrm{SL}}^{\mathrm{C}}$ can be defined for a stress state lying on the SL with the same Lode's angle as the actual state. The interpolation will give $\underline{\underline{A}}_{\mathrm{SL}}$ and $\underline{\mathbf{b}}_{\mathrm{SL}}^{\prime}$. However, this couple of tensors does not meet the consistency equation in general; the simplest way to adjust the interpolation scheme in order to meet the condition is to add a rotation of the tensor $\underline{\underline{A}}$,

$$
\underline{\mathbf{A}}_{\mathrm{SL}}=\underline{\mathbf{A}}_{\mathrm{SL}}^{0} \underline{\mathbf{Q}}
$$

with $\mathbf{A}_{S L}^{0}$, the result of the interpolation defined in (34) when applied to axisymetric image states lying on the limit surface

$$
\underline{\underline{A}}_{S L}^{0}=\theta^{\mathrm{B}} \underline{\underline{\mathbf{A}}}_{S \mathrm{~B}}^{\mathrm{B}}+\theta^{\mathrm{C}} \underline{\underline{\mathrm{A}}}_{\mathrm{SL}}^{\mathrm{C}}
$$

\footnotetext{
${ }^{\ddagger} \mathrm{SL}=$ surface limite; CLoE model's notations have been defined in french.
} 
The rotations do not modify the eigen values of the tensor but only the direction of the eigen vectors.

(3) Finally, knowing $\underline{\underline{A}}^{\mathrm{R}}$ and $\underline{\underline{\mathbf{A}}} \mathrm{sL}$, a weighting procedure using the reduced deviatoric stress $\bar{q}$ is used to define the current $\underline{\underline{\mathbf{A}}}$ tensor; $\underline{\underline{\mathbf{A}}}^{\mathrm{R}}$ will be coincident with $\underline{\underline{\mathbf{A}}} \mathrm{s}$ at limit surface $(\vec{q}=1)$ :

$$
\underline{\underline{\mathbf{A}}}^{\mathrm{R}}=(1-\bar{q}) \underline{\underline{\mathbf{A}}}^{\mathrm{R} \mathbf{1}}+\bar{q} \underline{\underline{\mathbf{A}}}_{\mathrm{SL}}
$$

The interpolation is not used for the three moduli denoted $g, h$ and $j$ in equation (8). Another interpolation is performed, based on one hand upon objectivity which implies some links between these moduli and other moduli of A - (see for instance equations (9) and $(10)$ - and on the other hand upon the results provided by a bifurcation analysis. Details will be explained in a next paper as explained in the introduction.

\section{CLOE FOR HOSTUN RF SAND}

\subsection{Identification}

As discussed in Section 4.8, CLoE is based on analytical formulations of the material responses on a set of basic paths. The computer implementation of the model uses a number of numerical parameters, which describe the analytical formulations: for example, the quantities $a_{h c}, c_{h c}, p_{f c}$ and others shown in Figures 5-7. However, input material data of CLoE are not these numerical parameters themselves, but a set of more physical input data. Reasons for that are both practical (the meaning of the parameters would be totally obscure for the user) and technical (some dependencies exist between the formulations of the responses on different paths, due to necessary consistency in the isotropic states).

In order to carry on this work, an identification procedure has been defined, which is considered as a part of the model. The data that it takes (interactively) are essentially familiar geotechnical data, like friction angle (compression and extension), dilatancy factor (the slope of the linear part of the $\left(\varepsilon_{\mathrm{v}}, \varepsilon_{1}\right)$ curve in axisymmetric tests, characteristic point (the transition point from contractancy to dilatancy), and a few more that can be determined easily from the experimental curves. Some input data are less intuitive, namely the pseudoisotropic ones; for these data, a reasonable value is proposed by the procedure, to be checked.

Finally the identification procedure outputs a set of numerical parameters, to be used as material parameters by the constitutive code package. The latter can be embedded in a FEM code or in any path-integration code.

The identification of shear moduli, which must be made in relation with localization observations, is not discussed in this paper. The examples presented here do not activate the shear moduli of the constitutive equations.

Figure 8 presents the successive steps of the interactive identification procedure for CLOE model parameters.

Step 1: Limit surface. The procedure starts with the specification of the limit surface parameters. Limit friction angles $\varphi_{\mathrm{C}}$ and $\varphi_{\mathrm{E}}$ mobilized in axisymmetric triaxial compression and extension tests are requested; it is the user's responsibility to define it on the basis of experimental data, knowing that the idealized response considered and modelized by CLOE does not take into account any post-peak softening material characteristics. The limit surface is defined by three constants $\alpha, \beta$ and $n$ according to equation (30). The default value 0.229 is given to $n$, but it can be 


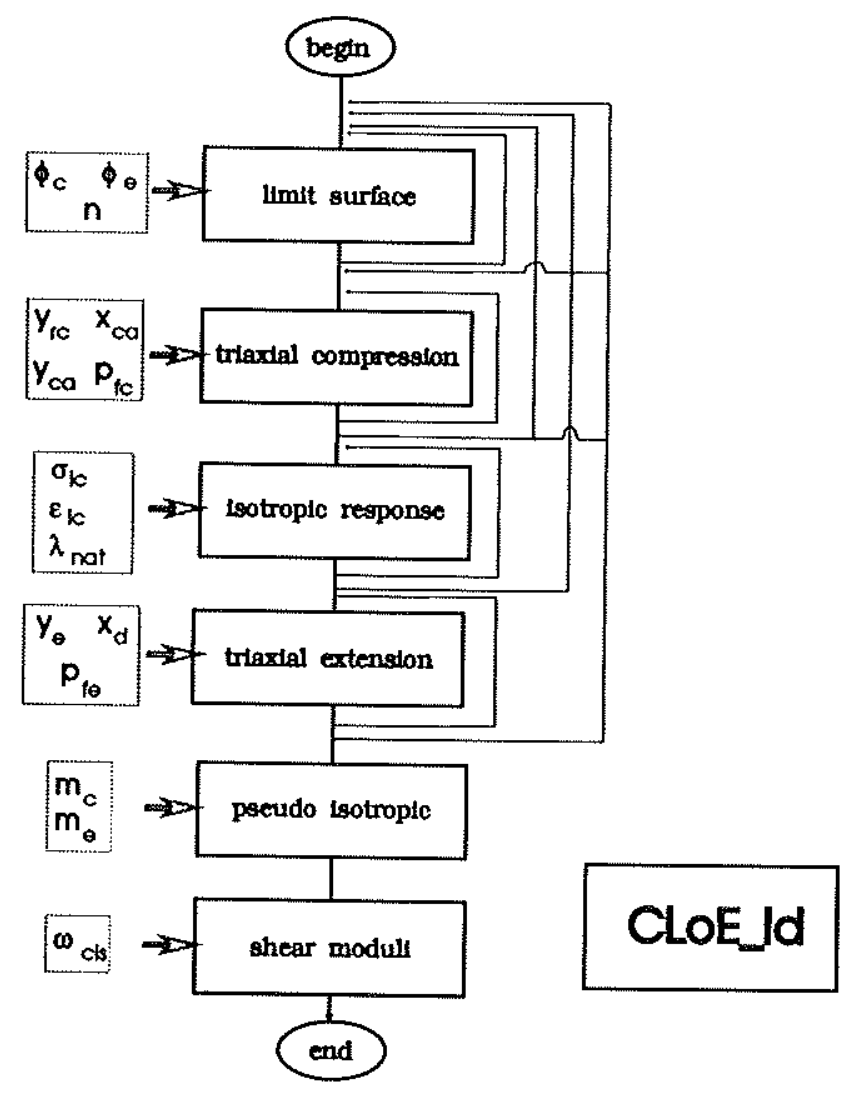

Figure 8. Successive steps of the interactive identification procedure for CLoE model parameters,

adjusted by the user. $\alpha$ and $\beta$ are given by the equations

with

$$
\begin{gathered}
\beta=\frac{\left(\bar{r}_{\mathrm{C}} / \bar{r}_{\mathrm{E}}\right)^{1 / n}-1}{\left(\bar{r}_{\mathrm{C}} / \bar{r}_{\mathrm{E}}\right)^{1 / n}+1} \\
\alpha=\frac{\bar{r}_{\mathrm{C}}}{(1+\beta)^{n}}
\end{gathered}
$$

$$
\begin{aligned}
& \bar{r}_{\mathrm{C}}=\sqrt{2} \frac{2 \sin \varphi_{\mathrm{C}}}{3-\sin \varphi_{\mathrm{C}}} \\
& \bar{r}_{\mathrm{E}}=\sqrt{2} \frac{2 \sin \varphi_{\mathrm{E}}}{3+\sin \varphi_{\mathrm{E}}}
\end{aligned}
$$

The convexity of the limit surface is checked by a simple procedure, leading to a modification request in case of convexity violation.

Step 2: Triaxial compression response. The second step of the procedure concerns the axisymmetric triaxial response. It is usually the path for which most reliable experimental data are available, sometimes the only one. Quantities $y_{r c}, x_{c a}, y_{c a}$ and $p_{f c}$ are requested from the user 
(defined in Figure 5): together with $\varphi_{\mathrm{C}}$ already known, this data set is sufficient to fix the analytical constants of the simple curves chosen a priori as the idealization of the material elementary response on axisymmetric triaxial path. The derivation of the coefficients of the stress ratio hyperbola, the volumetric strain parabola and final straight line is straightforward; the results are

$$
\begin{gathered}
c_{h c}=\frac{y_{r c}-1}{x_{c a}\left(y_{i n f c}-y_{r c}\right)} \\
a_{h c}=c_{h c}\left(y_{i n f c}-1\right)
\end{gathered}
$$

with

$$
y_{i n f c}=\tan ^{2}\left(\pi / 4+\varphi_{\mathrm{C}} / 2\right)
$$

For the parabola $\varepsilon_{\mathrm{v}}=a_{p c} \varepsilon_{a}^{2}+b_{p c} \varepsilon_{a}$, we have

$$
\begin{gathered}
a_{p c}=\frac{-y_{c a}}{x_{c a}^{2}} \\
b_{p c}=-2 a_{p c} x_{c a}
\end{gathered}
$$

and the transition to the straight line is at the abscissa $x_{p c}$;

$$
x_{p c}=\frac{p_{f c}-b_{p c}}{2 a_{p c}}
$$

To continue the procedure, extension triaxial idealized response parameters have to be identified. However, this cannot be done independent of the compression response. Consistency of the response when the stress state is isotropic requires that axisymmetric compression, extension and isotropic responses are linked in isotropic states, by three equations

$$
\begin{gathered}
\frac{1+v_{\mathrm{C}}}{E_{\mathrm{C}}}=\frac{1+v_{\mathrm{E}}}{E_{\mathrm{E}}} \\
\frac{1}{E_{\mathrm{C}}}-\frac{1}{E_{\mathrm{E}}}+k\left(\frac{\sqrt{1+2 v_{\mathrm{C}}^{2}}}{E_{\mathrm{C}}}+\frac{\sqrt{1+2 v_{\mathrm{E}}^{2}}}{E_{\mathrm{E}}}\right)=0 \\
E_{\mathrm{lc}}=E_{\mathrm{C}} \frac{1+k \sqrt{3}}{1-2 v_{\mathrm{C}}+3 k \sqrt{1+2 v_{\mathrm{C}}^{2}}}
\end{gathered}
$$

with $E_{\mathrm{C}}, E_{\mathrm{E}}$ and $E_{\mathrm{ic}}$ the stress-strain moduli, normalized by the isotropic stress, on compression, extension and isotropic paths and $v_{\mathrm{C}}, v_{\mathrm{E}}$ the Poisson's coefficient on compression and extension paths. $k$ is the modulus of the constitutive tensor $\underline{b}^{\prime}$ in isotropic state as stated in equation (10). The equations are derived by expressing the response of the model for unit stress rates on the different basic paths, and combining the resulting equations; the detailed derivation, too extensive to be given here, can be found in References 39 and 40 , available on demand to the authors.

Given $E_{\mathrm{C}}, v_{\mathrm{C}}$ and $E_{\mathrm{ic}}$, these equations allow us to determine $k$; in addition $E_{\mathrm{B}}$ and $v_{\mathrm{E}}$ are imposed.

Step 3: Isotropic response. The third step of the identification procedure concerns the isotropic response. It can be shown easily that, due to the hypothesis of stress level linear dependency (discussed in Section 4.7), the isotropic response is an exponential curve

$$
\sigma_{i}=\sigma_{\mathrm{ir}} \mathrm{e}^{\lambda_{c}\left(\varepsilon_{i}-\varepsilon_{\mathrm{ir}}\right)}
$$


with $\sigma_{i}$ and $\varepsilon_{i}$ the traces of the stress and strain tensors, $\lambda_{c}$ the (normalized) slope of the isotropic response curve in a reference point $\left(\sigma_{\mathrm{ir}}, \varepsilon_{\mathrm{ir}}\right), \lambda_{c}$ is said to be normalized because by derivation we get

$$
\dot{\sigma}_{i}=\lambda_{\mathrm{c}} \sigma_{\mathrm{ir}} \mathrm{e}^{\lambda_{c}\left(\varepsilon_{i}-\varepsilon_{\mathrm{rr}}\right)} \dot{\varepsilon}_{i}
$$

and at the reference point $\left(\sigma_{i}=\sigma_{\mathrm{ir}}, \varepsilon_{i}=\varepsilon_{\mathrm{ir}}\right)$ :

$$
\dot{\sigma}_{i}=\lambda_{c} \sigma_{\mathrm{ir}} \dot{\varepsilon}_{i}
$$

Hence

$$
\lambda_{c}=\frac{1}{\sigma_{\mathrm{ir}}} \frac{\dot{\sigma}_{i}}{\dot{\varepsilon}_{i}}
$$

which is also the definition valid for $E_{i c}$ mentioned above.

In the identification procedure, it has been chosen for user's convenience to request the reference stress and strain, and the corresponding slope $\lambda_{\text {nat }}$ in terms of cell pressure $p$ (one-third of the trace of the stress tensor) and volumetric strain $\varepsilon_{\mathrm{y}}$ (trace of the strain tensor).

$$
\lambda_{\text {nat }}=\frac{\dot{p}}{\dot{\varepsilon}_{\mathrm{y}}}
$$

The parameter for isotropic response will be

$$
\lambda_{\mathrm{c}}=E_{\mathrm{ic}}=\frac{1}{\sigma_{i \mathrm{r}}} \times 3 \times \lambda_{\mathrm{nat}}
$$

Step 4: Triaxial extension response. As discussed above, $E_{\mathrm{E}}$ and $v_{\mathrm{E}}$ are imposed by the consistency in isotropic states. The stress-strain hyperbola for triaxial extension is already entirely determined, given the initial modulus by $E_{\mathrm{E}}$ and the asymptotic by $\varphi_{\mathrm{E}}$. We get

$$
\begin{gathered}
a_{h e}=E_{\mathrm{E}} \\
c_{h e}=\frac{a_{h e}}{y_{\text {infe }}-1}
\end{gathered}
$$

with

$$
y_{\text {infe }}=\tan ^{2}\left(\pi / 4+\varphi_{\mathrm{E}} / 2\right)
$$

The constants of the analytical formulation of the volumetric strain response in triaxial extension are determined on the basis of experimental data requested from the user, and some default values: The parameters $x_{m 2}$ and $x_{\mathrm{c}}$ define the bounds of the initial cubic curve, and the subsequent straight line (Figure 6); they are assigned a default value by

$$
\begin{gathered}
x_{m 2}=x_{d} / 5 \\
x_{c}=x_{d} / 4
\end{gathered}
$$

where $x_{d}$, the intersect of the final straight line, is given by the user together with $p_{f e}$ the final dilatancy slope and $y_{e}$ the volumetric strain at the intermediate plateau. The following constants for the cubic and the quadratic parts of the curve can be obtained as

$$
\begin{gathered}
c_{1}=1-2 y_{\mathrm{E}} \\
b_{1}=\frac{3 y_{e}-2 c_{1} x_{m 2}}{x_{m 2}^{2}} \\
a_{1}=\frac{-c_{1}-2 b_{1} x_{m 2}}{3 x_{m 2}^{2}}
\end{gathered}
$$


for cubic and

$$
\begin{gathered}
c_{b}=y_{e} \\
x_{t 2}=\frac{4}{3}\left(\frac{y_{e}}{p f_{e}}-\frac{x_{c}}{4}+x_{d}\right) \\
a_{b}=\frac{p f_{e}}{4\left(x_{t 2}-x_{c}\right)^{3}}
\end{gathered}
$$

for quadratic.

Step 5: Pseudoisotropic response. The pseudoisotropic is not a common path; users are usually lacking any experimental data for that path. The identification procedure assigns the default values $m_{\mathrm{E}}=0$ and $m_{\mathrm{C}}=1$.

Step 6: Shear moduli. The shear moduli are controlled by a parameter whose identification has to be done in connection with shear band analysis. This is not in the scope of this paper. The identification procedures simply request the value of this parameter, and proposes a default value.

\subsection{Illustration of the response}

Figures 9 (a) - (c) show the responses for axisymmetric triaxial undrained monotonous loading on dense and loose Hostun RF sand (dense sand is denoted by dots on the curves). Figure 9(a) indicates that the dense sand tends to follows a linear path - slightly divergent from the trace of the limit surface-in the $p, q$ plane, while the loose sand tends to an accumulation point in this plane; this is coherent with the continued dilation considered in the model for dense sands, which has been dropped to zero in the loose sand set of parameters. Figure $9(b)$ shows that the deviatoric stress $q$ tends to an asymptote in the loose case, while in the dense it increases continuously; again
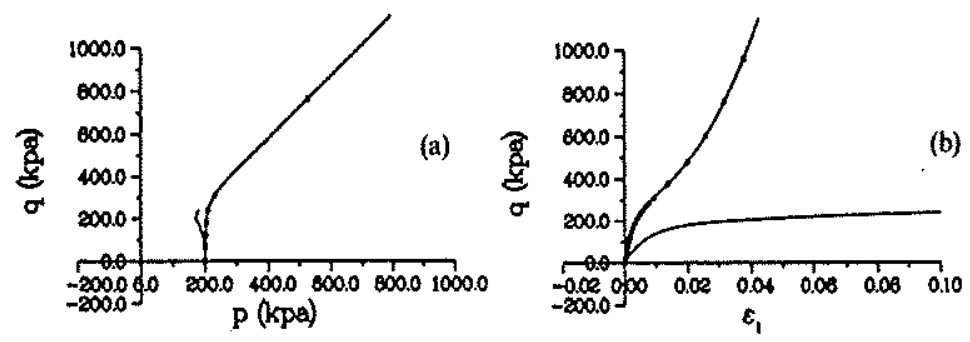

\begin{tabular}{|ll|}
\hline symbole & sable \\
\hline- -*- & dense, jeu 1 \\
- & lâche, jeu 2 \\
\hline
\end{tabular}

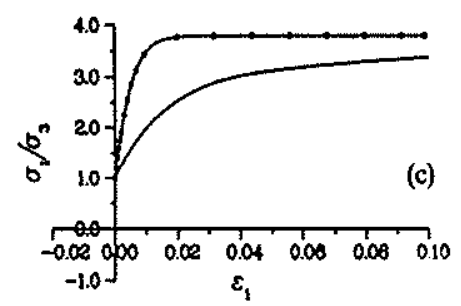

Figure 9. Illustration of the response of the model on homogeneous stress strain paths: undrained axisymmetric triaxial compression responses for a dense (dotted lines) and a loose sand. This path is not a formulation path, so it is a real test of the model integration. 


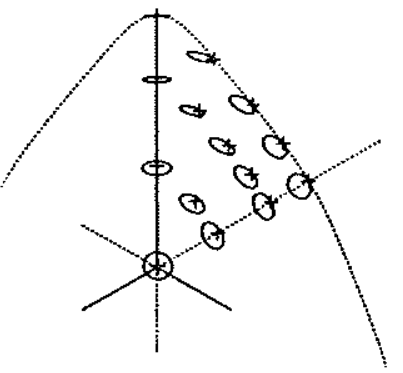

plan déviatoire

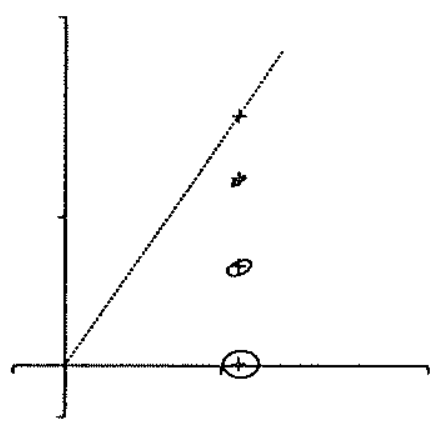

plan $q, p$ à $\phi=0^{0}$

Figure 10. Gudehus diagrams at various stress states - consistency check. One can verify that the consistency requirement is met, i.e. the envelops do not cross the limit surface.

this is due to the dilatancy in the dense sand, which induces a continuous drop of the pore pressure, and hence an increase of the effective isotropic stress; in real world experiments, this phenomenon is limited by cavitation in the pore fluid, but this is not considered here. Figure 9(c) shows the fact that both dense and loose sands tend to a (different) state in terms of effective stress ratio, or equivalently in term of mobilized friction angle.

More illustrative of the specificity of CLoE are Figures 10(a) and (b). Here the constitutive responses at different stress states, from isotropic one to limit states are shown. We use the representation introduced by Gudehus: ${ }^{30}$ the egg-shaped diagrams associated with a cross give a section of the three-dimensional envelope of stress rate vectors mapping the response to a unit strain rate vector's sphere.

First we can observe that, although highly non-linear, the response envelopes remain continuous in any state. Second, these figures show that the consistency condition at the limit surface is met, since the stress rate envelops for limit stress states are tangent to the limit surface. This can be seen in the deviatoric plane, and in the $p, q$ plane.

Some aspects of Figure 10 deserve a comment. The Gudehus diagram associated with the compression triaxial stress state belonging to the limit surface seems to degenerate. Plotted with another scale, however, it is seen that this diagram does not degenerate. However, it seems to predict a very soft behaviour at unloading. The explanation is rather simple and shows that interpretation of such diagrams is not always easy. For compression unloading a very contractant behaviour has been assumed. In this case the unit strain rate corresponds to a very little variation of the strain component associated with the unloading direction. This is the reason why the dimensions of the corresponding Gudehus diagram are so small. Let us notice that this is not the case for corresponding extension state as in our example, such a large contractancy was not assumed for extension unloading.

\section{CONCLUSION}

The examples presented above show that $C L O E v 1.00$ fullfils the basic requirements of the project: to be a reasonable representation of sand behaviour, and to remain strictly consistent when reaching the limit admissible states. Later experience with this new model, including parameter sensibility check and bifurcation tests, will give a firm basis for practical modelling of soil engineering problems. Extensive experience of integration of that model in FEM code 
$L A G A M I N E$, University of Liège, has led to very encouraging results: CLoE is showing very good robustness, reasonably low CPU time expenses, and efficient localization criterion testing procedure. This experience will be reported in an forthcoming paper.

\section{REFERENCES}

1. R. Hill, The Mathematical Theory of Plasticity, Oxford Univ. Press, 1950.

2. R. Chambon and B. Renoud-Lias, 'Incremental non linear stress-strain relationship for soils and integration by FEM', in Numerical Methods in Geomechanics, Vol. 1, Balkema, 1979, pp. 405-413.

3. R. Chambon, 'Contribution à la modélisation numérique non linéaire des sols', Thèse de doctorat es sciences, USMG-INPG, 1981.

4. P. Royis, Formulation mathématique de lois de comportement, modélisation numérique de problemes aux limites en mécanique des solides deformables Univ. de Grenoble, 1986.

5. G. Gudehus, Mechanics of geomaterials, chapter Requirements for constitutive relations for soils, Wiley, 1985, pp. $47-63$.

6. J. Desrues and R. Chambon, 'Shear band analysis for granular materials: the question of incremental non linearity', Ingenieur Archiv, 59, 187-196 (1989).

7. J. Lanier, 'Etude expérimentale des lois de comportement en grandes déformation à l'aide d'une presse réellement tridimensionnelle', Cahiers du groupe français de rhéologie, $4(2)$, pp. 53-60 (1976).

8. A. S. Saada, 'Hollow cylinder torsional device: their advantages and limitations', Advanced Triaxial Testing for Soil and Rocks-ASTM STP 977, ASTM, 1988, pp. 766-795.

9. J. R. F. Arthur, 'Cubical devices: versatility and constraints', Advanced Triaxial Testing for Soil and Rocks-ASTM STP 977, ASTM, 1988, pp. 743-765.

10. H. Joer, J. Lanier, J. Desrues and E. Flavigny, '1g2e: a new shear apparatus to study the behaviour of granular materials', Geotechnical Testing J. ASTM 15(2), 129-137 (1992).

11. J. R. F. Arthur and B. K. Menzies, 'Inherent anisotropy in a sand', Géotechique, 22(1), 115-128 (1972),

12. J. R. F. Arthur, K. S. Chua and T. Dunstan, 'Induced anisotropy in a sand', Géotechnique, 22(1), 13-30 (1977).

13. A. W. Bishop and G. E. Green, 'The influence of end resiraint on the compression strength of a cohensionless soil', Géotechnique, 15(3), 243-266 (1965).

14. W. M. Kirkpatrick and D. J. Belshaw, 'On the interpretation of the triaxial test', Géotechnique, 18(3), 336-350 (1968),

15. J. L. Colliat-Dangus, J. Desrues and P. Foray, 'Triaxial testing of granular soil under elevated cell pressure', Advanced Triaxial Testing for Soil and Rocks-ASTM STP 977, ASTM, 1988, pp. 290-310.

16. J. Desrues, M. Mokni and F. Mazerolle, 'Tomodensitométrie et localisation dans les sables', in Comptes-rendus du $X$ ECSMFE-Florence mai $91,1991$.

17. J. Desrues, R. Chambon, M. Mokni and F. Mazerolle, 'Void ratio evolution inside the shear bands in triaxial sand samples studied by ct technique', $3 d$ Int. Workshop in Localisation and Bifurcation for Soils and Rocks-Aussois 6-9 September 93, 1993.

18. A. Dresher and I. Vardoulakis, 'Geometric softening in triaxial tests on granular material', Géotechnique, 32(4), 291-303 (1982).

19. R. Hill, 'Acceleration waves in solids', J. Mech. Phys. Solids, 10, 1-16 (1962).

20. J. Mandel, Rhéologie et Mécanique des Sols, chapter Conditions de stabilité et Postulat de Drucker, pp. 58-68. 1964.

21. J. R. Rice, 'The localisation of plastic deformation', in Theoretical and Applied Mechanics, North Holland, 1976.

22. I. Vardoulakis, 'Shear band inclination and shear modulus of sand in biaxial tests', Int. J. Num. Anal. Meth. Geom., 4, 103-119, 1980.

23. R. Chambon and J. Desrues, 'Bifurcation par localisation et non linéarité incrémentale: un exemple heuristique d'analyse complète', Plastic Instability, Presses ENPC Paris, 1985, pp. $101-119$.

24. B. Loret, 'Non-linéarité incrémentale et localisation des déformations: quelques remarques', J. Mécanique Théor, Apliqué, 6(3), 423-459 (1987).

25. D. Kolymbas, 'An outline of hypoplasticity', Arch. appl. mech. 61, 143-151 (1991).

26. R. Hill, 'Some basic principles in the mechanics of solids without a natural time', J. Mech. Phys. Solids, 7, 209-225 (1959).

27. Z. P. Bazant, 'Endochronic inelasticity and incremental plasticity'. Int. J Solids Struct. 14, 691-714 (1978).

28. F. Darve, M. Boulon and R. Chambon, 'Loi rhéologique incrémentale des sols', J. Mécanique, 17(5), 679-716 (1978).

29. I. Nelson, 'Constitutive models for use in numerical computations', Plastic and Long Term Effects, A. A. Balkema, 1977.

30. G. Gudehus, 'A comparison of some constitutive laws for soils under radially symmetric loadings and unloadings', in W. Wittke (ed.) Numerical Methods in Geomechanics, Balkema, 1979, pp. 1309-1323.

31. R. Chambon, 'Une loi théologique incrémentale non linéaire pour les sols non-visqueux, J. M. T. A., 3(4), 521-544 (1984).

32. J. Desrues, 'La localisation de la déformation dans les matériaux granulaires', These de Doctorat es Sciences, USMG et INPG, 1984 . 
33. D. Kolymbas, 'A novel constitutive law for soils', Constitutive Laws for Ingeneering Materials, Elsevier New York, 1987, pp. 319-326.

34. J. Lanier, 'Etude d'une loi de comportement pour des matériaux non visqueux', J. Mécanique, 18(1), 175-195 (1979).

35. K. C. Valanis, 'A theory of viscousplasticity without a yield surface', Arch. mech. 23, 517-551 (1971).

36. P. V. Lade and J. M. Duncan, 'Elastoplastic stress-strain theory for cohesionless soil', Proc. ASCE, 101, 1037-1053 (1975).

37. H. Matsuoka and T. Nakai, 'A new failure criterion for soils in three-dimensional stresses', in Proc. IUTAM Conf. Def. Fail. Gran. Media, Delft (NL), 1982, pp. 253-263.

38. H. A. M. van Eekelen, 'Isotropic yield surface in three dimensions for use in soil mechanics, Int. J. Num. Anal. Meth. in Geom., 4, 89-101 (1980).

39. R. Chambon, J. Desrues, W. Hammad and R. Charlier, 'CLoE, Consistance et Localisation Explicite: une loi incrémentale non linéaire', Rapport interne du Groupe de Géomécanique IMG, 1990.

40. W. Hammad, 'Modélisation non linéaire et étude expérimentale de la localisation dans les sables', Thèse de doctorat, UJF-INPG, 1991. 